\title{
DYNAMICS ANALYSIS OF FLAPPING WING ACTUATOR
}

\author{
Janis Viba, Valters Cipruss, Oskars Ozolins, Janis Gulbis \\ Riga Technical University, Latvia \\ janis.viba@rtu.lv, valtersc@inbox.lv, oskars.ozolins@rtu.lv, janis.gulbis@rtu.lv
}

\begin{abstract}
In this paper various simple plane configurations of the wings of the actuators by motion investigations in fluid (water or air) are being reviewed. The first part of the report deals with the investigation of motion of a single degree of freedom (1DOF) - rotation system. The rotational motion equations are obtained by the relations of classical mechanics. An approximate one-sided interaction of the fluids and wing elements is described by the exchange the momentum of the fluid particle flux impact. Approximate correlations are obtained for modeling the rotation motion with different actuator excitations. The possibilities of adding a rotary linear or non - linear spring are analyzed. The possibility of blocking the actuator's movement with a stop is considered. In the second part of the report, plane motion of the object is analyzed taking into account the results of the first part of investigations. An analysis is carried out to synthesize the actuator's application for objects flying or diving motion excitation in robot technics.
\end{abstract}

Keywords: fluid interaction, blade actuators, vibrations.

\section{Introduction}

Vibrating motors play an important role in energy handling in the vibro technics, vibro technology, automation and robotics. Among these engines, for object motion control inside fluid (in water, in the air), flapping wing actuators are applied. For example, the theoretical framework of an electromagnetic actuator with the intended application to flapping-wing vehicles is presented and validated through experimental data [1]. Flying insects and robots that mimic them flap and rotate (or 'pitch') their wings with large angular amplitudes are analyzed in [2]. Herein, detailed equations of motion are derived for wing rotational dynamics, and a blade-element model is used to supply the aerodynamic force and moment estimates. The fixed, flapping and rotary wing aerodynamics for micro air vehicle applications are described in the book [3].

Dynamics calculation of flappy wing actuators includes two important aspects to consider: wing motion is in small Reynolds number region (as Stokes flow); in vibration interactions relative velocities of flow particles are changing directions against wing normal direction (because the wing movement is composed of translation and rotation). Examples of such research direction could include the works [4-9].

In this work, the first model looks at the actuated wing, which rotates or vibrates around a motionless horizontal axis (Fig. 1). It is assumed that the fluid flow $V F$ is parallel to the vertical yaxis.

The rotational motion equations are obtained by the relations of classical mechanics [10; 11]. An approximate one-sided interaction of the fluids and wing elements is described by exchange of the momentum of the fluid particle flux impact. Approximate correlations are obtained for modelling the rotation motion with different actuator excitations. The possibilities of adding a rotary linear or non linear spring are analysed. The possibility of blocking the actuator's movement with a stop is considered.

An analysis was carried out to synthesize the actuator's application for energy recovery from fluid (air or water). The obtained fluid and wing interaction forces in the subsequent study are used to solve more complex tasks.

\section{Analysis of rotating wing}

The rotational motion model is shown in Fig. 1. It includes a fixed horizontal axis $\mathrm{O}$, around which a blade of length $\mathrm{L}$ can rotate. The flow velocity VF is taken upright and parallel to y-axis. In the simplified model considered here, the main interaction force Rn between the wing and the fluid is perpendicular to the surface (called as lifting drag or lifting interaction). The classical mechanics formulas are used to calculate the interaction force $[10 ; 11]$. According to the theory of fluid interaction it is assumed that the elementary local forces are proportional to the relative speed in the 
square. In the center $\mathrm{O}$ the main vector $R n$ of interactions and the central moment $M O$ can be calculated using formulas (1) and (2) $[11,14,15]$ :

$$
\begin{gathered}
R n=Q 0 \cdot\left\{\int_{L 0}^{L 0+L}\left[(\xi \cdot \omega+V F \cdot \cos (\varphi))^{2}\right] \cdot d \xi\right\} ; \\
M O=Q 0 \cdot\left\{\int_{L 0}^{L 0+L}\left[(\xi \cdot \omega+V F \cdot \cos (\varphi))^{2} \cdot \xi\right] \cdot d \xi\right\} ; \\
Q 0=\frac{D 0 \cdot \rho \cdot A 0}{2},
\end{gathered}
$$

where $\quad D 0-$ drag coefficient parameter;

$\rho$ - fluid density;

$A 0$ - middle value of area;

$V F$ - flow velocity $[12 ; 13]$.

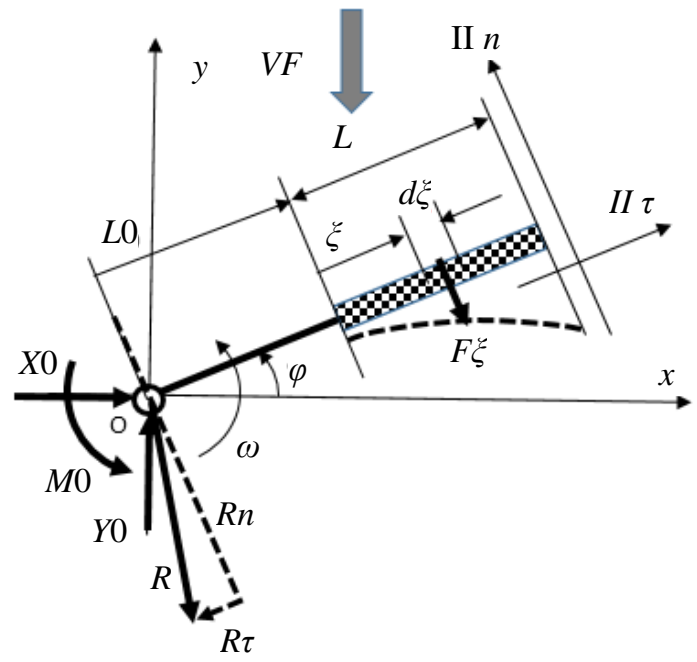

Fig. 1. Rotating wing model: $O$ - rotation axis; $L$ - length of wing; $L 0$ - distance till wing; $V F$ - flow velocity; $\xi$-integration coordinate; $d \xi$ - small interaction length; $F \xi$ - local small force; $\tau, n$-relative coordinates; $X 0, Y 0$ - reactions in axis $\mathrm{O} ; R n, R \tau$ - fluid interactions components; $M 0$ - fluid interactions moment; $\varphi, \omega$ - wing angle and angular velocity

After integration of equation (1) formulas $(3,4)$ can be found:

$$
\begin{gathered}
R n=Q 0 \cdot L \cdot\left[\begin{array}{l}
\frac{L^{2} \cdot \omega^{2}}{3}+L \cdot L 0 \cdot \omega^{2}+L \cdot V F \cdot \omega \cdot \cos (\varphi)+L 0^{2} \cdot \omega^{2}+ \\
+2 \cdot L 0 \cdot V F \cdot \omega \cdot \cos (\varphi)+V F^{2} \cdot(\cos (\varphi))^{2}
\end{array}\right] ; \\
M O=Q 0 \cdot\left[\begin{array}{l}
\frac{(L+L 0)^{4} \cdot \omega^{2}}{4}-\frac{L 0^{4} \cdot \omega^{2}}{4}+\frac{V F^{2} \cdot(\cos (\varphi))^{2} \cdot(L+L 0)^{2}}{2}-\frac{V F^{2} \cdot(\cos (\varphi))^{2} \cdot L 0^{2}}{2}+ \\
+\frac{2 \cdot V F \cdot \omega \cdot \cos (\varphi) \cdot(L+L 0)^{3}}{3}-\frac{2 \cdot L 0^{3} \cdot V F \cdot \omega \cdot \cos (\varphi)}{3}
\end{array}\right] .
\end{gathered}
$$

Parameters from equations (3) and (4) can be used for calculations of the wing rotation angle $\varphi$, angular velocity $\omega$ and reactions $X 0, Y 0$. Using formulas (3) and (4) directions of forces can be additionally checked. For this purpose, the "sign" operator is used, which depends on the angular velocity or angle. Some examples for small angles $\left(-90^{\circ}<\varphi<90^{\circ}\right)$ are given below. 


\section{Analysis of rotating wing with spring and harmonica excitation}

For wing rotation motion calculation, when the length parameter $L 0=0$, differential equation is (5) :

$$
J z \cdot \ddot{\varphi}=-Q 0 \cdot\left[\frac{L^{4} \cdot \dot{\varphi}^{2}}{4}+\frac{2 \cdot L^{3} \cdot V F \cdot \dot{\varphi} \cdot \cos (\varphi)}{3}+\frac{L^{2} \cdot V F^{2} \cdot(\cos (\varphi))^{2}}{2}\right]-c \cdot \varphi+M z \cdot \sin (p \cdot t)-b \cdot \dot{\varphi},
$$

where $J z$-moment inertia of wing;

$\varphi, \dot{\varphi}, \ddot{\varphi}-$ angle, angular velocity and angular acceleration;

$c$ - rotational spring stuffiness;

$b$ - additional damping constant;

$M z, p$ - parameters of harmonica excitation;

$V F$ - flow velocity;

$L$ - wing length.

Some results of calculation are given in Fig. 2, 3.

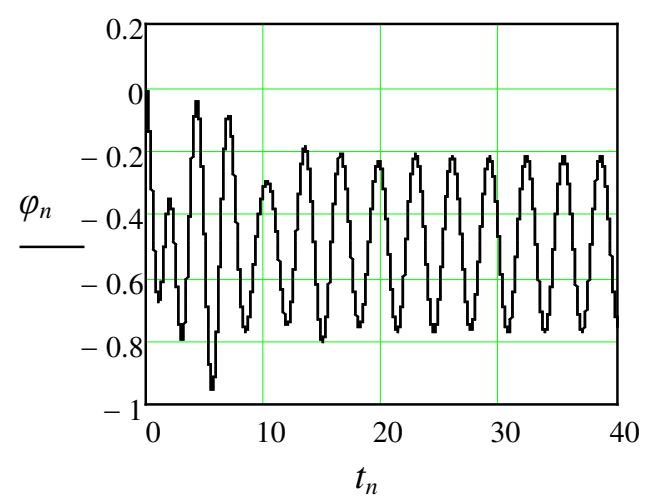

Fig. 2. Wing angle as time $t$ function in system

SI: $\varphi$ - angle, which is negative because flow velocity $V F$ is negative (indirect y axe)

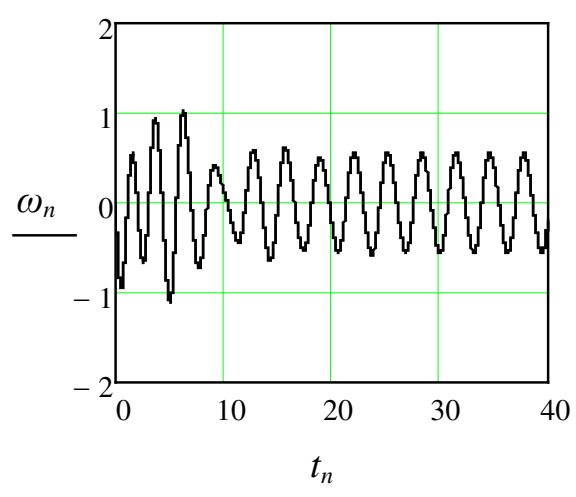

Fig. 3. Wing angular velocity $\omega$ as time $t$

function: $\omega$ - middle value of parameter is 0 because vibrations are around static equilibrium

\section{Analysis of rotating wing with spring and adaptive moment excitation}

Differential equation (5) for wing rotation motion calculation transforms to (6):

$$
J z \cdot \ddot{\varphi}=-Q 0 \cdot\left[\frac{L^{4} \cdot \dot{\varphi}^{2}}{4}+\frac{2 \cdot L^{3} \cdot V F \cdot \dot{\varphi} \cdot \cos (\varphi)}{3}+\frac{L^{2} \cdot V F^{2} \cdot(\cos (\varphi))^{2}}{2}\right]-c \cdot \varphi+M z \cdot \operatorname{sign}(\omega)-b \cdot \dot{\varphi},
$$

where $\operatorname{sign}(\omega)$ - means that the excitation moment $M Z$ changes the direction, when the angular velocity is changing.

Some results of calculation are given in Fig. 4, 5.

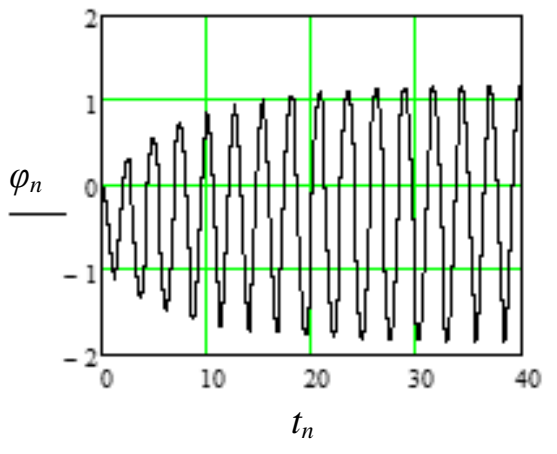

Fig. 4. Wing angle: $\varphi$ - angle, which is negative because flow velocity $V F$ is negative and exists new static equilibrium

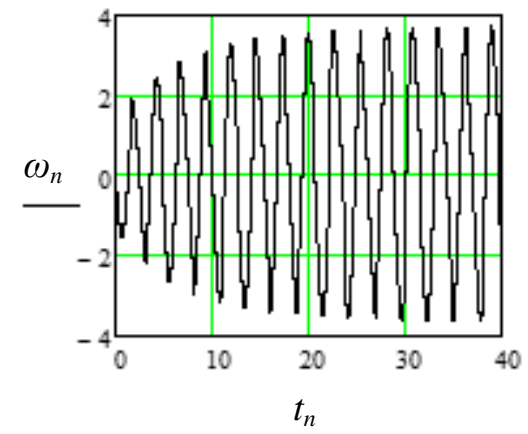

Fig. 5. Wing angular velocity: $\omega-$ middle value of parameter is 0 because vibrations are around static equilibrium 
Analysis of rotating wing with linear spring and adaptive drag exchange

In this case, differential equation (5) for motion calculation transforms to (7):

$J z \cdot \ddot{\varphi}=-Q 0 \cdot\{1-k \cdot \operatorname{sign}(\omega)\} \cdot\left[\frac{L^{4} \cdot \dot{\varphi}^{2}}{4}+\frac{2 \cdot L^{3} \cdot V F \cdot \dot{\varphi} \cdot \cos (\varphi)}{3}+\frac{L^{2} \cdot V F^{2} \cdot(\cos (\varphi))^{2}}{2}\right]-c \cdot \varphi-b \cdot \dot{\varphi},(7)$

where $k$-constant;

$\operatorname{sign}(\omega)$ - means that the drag coefficient parameter (2) is changing when the angular velocity is changing.

Some results of calculation are given in Fig. 6, 7.

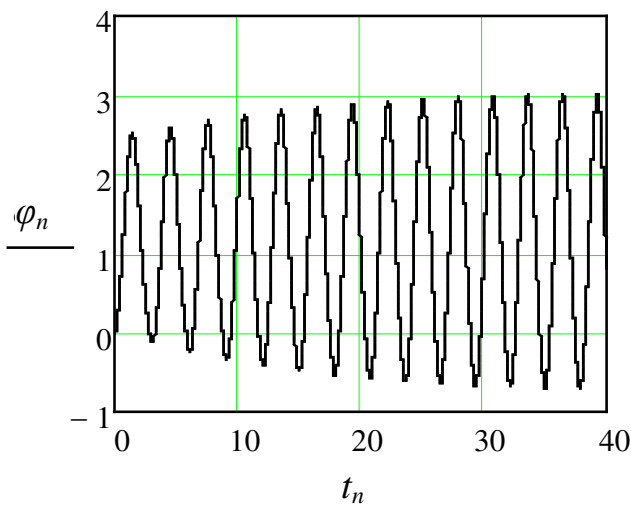

Fig. 6. Wing angle $\varphi$ as time $t$ function

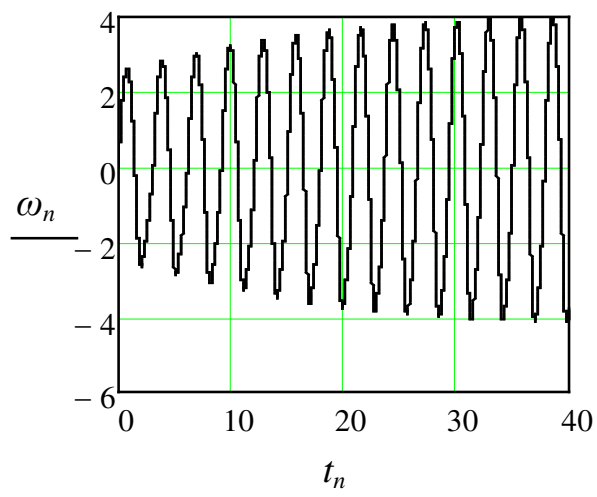

Fig. 7. Wing angular velocity $\omega$ as time $t$ function

Analysis of rotating wing with non-linear spring or obstacle impact and adaptive drag exchange

For these cases motion in the phase plane is shown in Fig. 8, 9. Analysis of motion shows that there are very large possibilities to synthesize different control actions for efficient motion control.

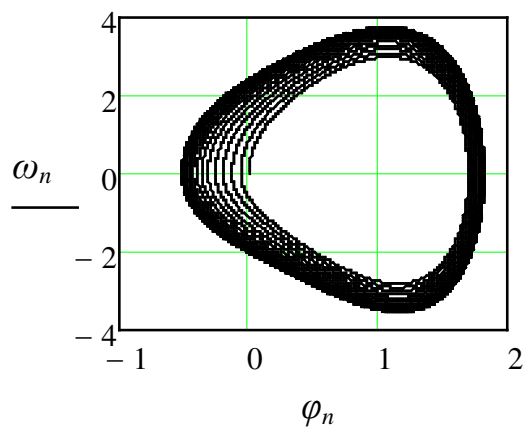

Fig. 8. Motion in phase plane with non - linear spring

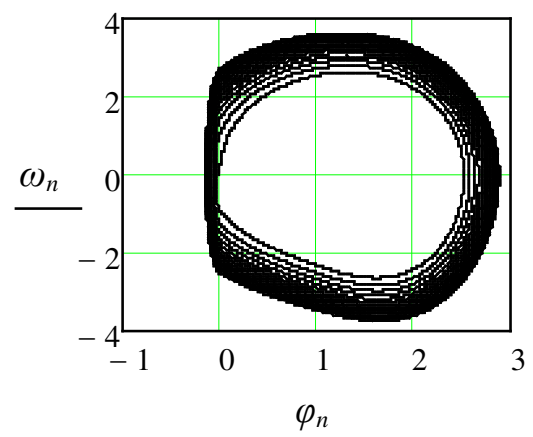

Fig. 9. Motion in phase plane with spring and impact

\section{Analysis of object motion with flapping wings}

Differential equations of object 1 vertical motion (Fig. 10) with flapping wing can be found by formulas of classical mechanics $[10,11,14,15]$. In this case, for the system of two degrees of freedom $(X 1, \varphi)$ two equations $(8)$ can be found:

$$
\begin{aligned}
& -(m 1+m 2) \cdot(g+\ddot{Y} 1)-m 2 \cdot L c \cdot\left[-\sin (\varphi) \cdot(\dot{\varphi})^{2}+\cos (\varphi) \cdot \ddot{\varphi}\right]-R n \cdot \cos (\varphi)-b 1 \cdot \ddot{Y} 1=0 ; \\
& -m 2 \cdot(g+\ddot{Y} 1) \cdot L c \cdot \cos (\varphi)-J z \cdot \ddot{\varphi}-c \cdot \varphi-R n \cdot L c-b 2 \cdot \dot{\varphi}+M z=0,
\end{aligned}
$$

where $m 1, m 2-$ object and wings mases;

$\ddot{Y} 1, \dot{Y} 1$ - vertical acceleration and velocity of body 1 ; 
$\varphi, \dot{\varphi}, \ddot{\varphi}$ - angle, angular velocity and angular acceleration of symmetric wings;

$L c$ - position of one wing centre mass;

$g$ - acceleration of free fall;

$b 1, b 2$ - damping parameters;

$M z$ - actuators moments.

Here it can be mentioned that in formulas (3-5) for $R n$ calculation $V F=\dot{Y} 1$ can be used. An example of modelling motion by Working Model 2D is shown in Fig. 11.

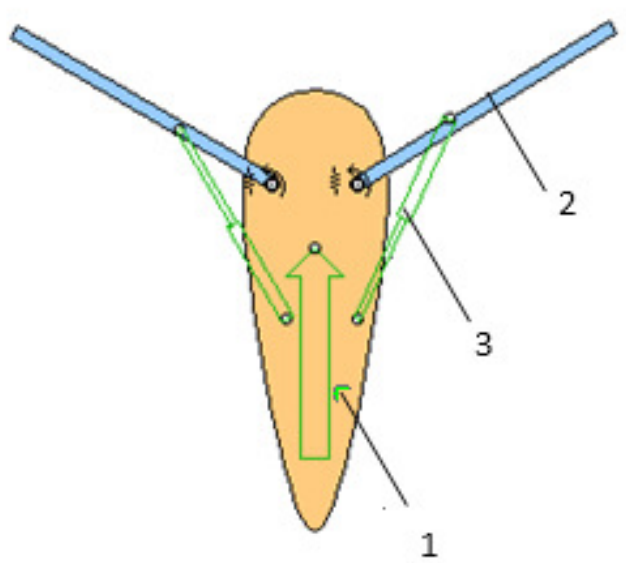

Fig. 10. Object vertical motion model: 1 central body; 2 -wing system; 3 - internal actuators

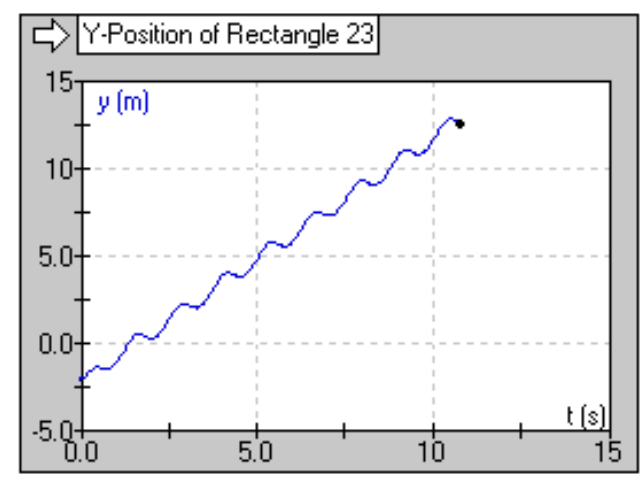

Fig. 11. Central body (mass m1) vertical displacement as time $t$ function

\section{Results and discussion}

Fluid and wing interactions obtained in this work (3) - (8) can be used to solve many engineering tasks of analysis, optimization and synthesis of different mechatronics systems with flapping wings. For example, for analysis and synthesis of the actuator's application for energy recovery from fluid (air or water) [16]. In flying robot motion control it is possible to solve tasks of robot motion up. Additionally, horizontally motion can be analyzed till given distance and return back to the starting position. For diving robot model motion investigations it is possible to analyze motion down and up, using "Archimedes force" control in equation (8). Movement through the frozen water reservoir below the ice surface can be investigated. In this task the dry friction coefficient between the object and ice can be taken into account. Part of this task will be investigated by the authors in future.

\section{Conclusions}

1. In this paper, a new approximation method describing the interaction of flapping wings with the rotation and composite motion in fluid flows has been developed.

2. A new area exchange control action idea has been given for the wing propulsion movement.

3. It is explained how the obtained relationships are supplemented by mechanisms with nonlinear elastic forces and adaptive feedback control.

4. The obtained mathematical relationships can be used to control and optimize mechatronics systems.

\section{References}

[1] Roll Jesse A., Cheng Bo., Deng Xinyan. An Electromagnetic Actuator for High-Frequency Flapping-Wing Microair Vehicles. IEEE Transactions on Robotics, April 2015, Vol .31(2), pp. 400-414. 
[2] Whitney J. P., Wood R. J. Aeromechanics of passive rotation in flapping flight. Journal of Fluid Mechanics, vol. 660, 2010. pp. 197-220.

[3] Mueller T.J. Fixed and Flapping Wing Aerodynamics for Micro Air Vehicle Applications. American Institute of Aeronautics and Astronautics. 2000-01-01. 595 p. [online][19.03.2018] Available at: http://ebookcentral.proquest.com/lib/rtulv-ebooks/detail.action?docID=3111539.

[4] Cho D.S., Kim B.H., Vladimir N., Choi T.M. Natural vibration analysis of rectangular bottom plate structures in contact with fluid. Ocean Engineering, 15 July 2015, Vol.103, pp.171-179.

[5] Pang M.J., Wei J.J. Analysis of drag and lift coefficient expressions of bubbly flow system for low to medium Reynolds number. Nuclear Engineering and Design, 2011, Vol. 241(6), pp. 2204-2213.

[6] Keivan F., Abasali F., Ehsan F., Sedaghati Z.N., Atena G. Numerical simulation of planar shear flow passing a rotating cylinder at low Reynolds numbers. Acta Mechanica, Feb, 2012, Vol. 223(2), $221 \mathrm{p}$.

[7] Kim J., Lee S. Modeling drag force acting on the individual particles in low Reynolds number flow. Powder Technology, July 2014, Vol.261, pp .22-32.

[8] Jeong J.T. Axisymmetric Stokes flow due to the motion of a thin disk along the axis of a circular tube. European Journal of Mechanics / B Fluids, January-February 2018, Vol.67, pp.397-403.

[9] Box F., Han E., Tipton C., Mullin T. On the motion of linked spheres in a Stokes flow. Experiments in Fluids, 2017, Vol. 58(4), pp. 1-10.

[10] Goldstein Herbert, Poole Charles, Safko John. Classical Mechanics. 3rd edition. Harlow: Pearson, 2014. 638 p. ISBN 978-1-292-02655-8.

[11] Meriam J. L., Kraige L. G., Bolton J. N. Engineering Mechanics: Dynamics, 8th Edition._Wiley. 2015. $736 \mathrm{p}$.

[12] Blevins Robert D. Applied Fluid Dynamics Handbook. Malabar, Fla.: Krieger, 2003. 570 p. ISBN 978-1-57524-182-1.

[13] Munson Bruce R., Rothmayer Alric P., Okiishi Theodore H., Huebsch Wade W. Fundamentals of Fluid Mechanics. 7th edition. Hoboken, NJ : Wiley, 2012. 792 p. ISBN 978-1-118-11613-5.

[14] Viba J., Eiduks M., Irbe M. Double pendulum vibration motion in fluid flow. In: Proceedings of International Conference "Engineering for Rural Development 2015". Jelgava, Latvia University of Agriculture; 2015, pp. 434-439.

[15] Viba J., Beresnevich V., Noskovs S., Irbe M. Investigations of rotating blade for energy extraction from fluid flow. In: Vibroengineering Procedia. Kaunas, Lithuania: JVE International Ltd; 2016; 8, pp. 312-315.

[16] Viba J., Noskovs S., Beresnevich V.. Patent LV 15038, Republic of Latvia, Int.Cl. F03D7/06. Method for control of operation condition of wind turbine and device for its realization. Date of Patent 20.12.2015. 